\title{
The Sturm-Liouville Hierarchy of Evolution Equations and Limits of Algebro-Geometric Initial Data
}

Russell JOHNSON ${ }^{\dagger}$ and Luca ZAMPOGNI $\ddagger$

${ }^{\dagger}$ Dipartimento di Sistemi e Informatica, Università di Firenze, Italy

E-mail: johnson@dsi.unifi.it

$¥$ Dipartimento di Matematica e Informatica, Università degli Studi di Perugia, Italy

E-mail: zampoglu@dmi.unipg.it

Received October 17, 2013, in final form February 27, 2014; Published online March 05, 2014

http://dx.doi.org/10.3842/SIGMA.2014.020

Abstract. The Sturm-Liouville hierarchy of evolution equations was introduced in $[A d v$. Nonlinear Stud. 11 (2011), 555-591] and includes the Korteweg-de Vries and the CamassaHolm hierarchies. We discuss some solutions of this hierarchy which are obtained as limits of algebro-geometric solutions. The initial data of our solutions are (generalized) reflectionless Sturm-Liouville potentials [Stoch. Dyn. 8 (2008), 413-449].

Key words: Sturm-Liouville problem; $m$-functions; zero-curvature equation; hierarchy of evolution equations; recursion system

2010 Mathematics Subject Classification: 37B55; 35Q53; 34A55; 34B24

\section{Introduction}

It is well-known that the Korteweg-de Vries equation

$$
\begin{aligned}
& \frac{\partial u}{\partial t}=6 u \frac{\partial u}{\partial x}-\frac{\partial^{3} u}{\partial x^{3}}, \quad u=u(t, x), \\
& u(0, x)=u_{0}(x)
\end{aligned}
$$

can be solved for various classes of initial data $u_{0}(\cdot)$ by making systematic use of the fact that it is formally equivalent to the Lax equation

$$
\frac{d L_{t}}{d t}=\left[P, L_{t}\right]
$$

where $L_{t}$ is the Schrödinger operator

$$
L_{t}=-\frac{d^{2}}{d x^{2}}+u(t, x)
$$

and $P$ is the antisymmetric operator

$$
P=-4\left[D^{3}-\frac{3}{4}(D u+u D)\right], \quad D=\frac{d}{d x} .
$$

While this observation does not in and of itself provide a solution of (1), it does imply that, if $u(t, x)$ is a decent solution of $(1)$, then the spectrum of the operator $L_{t}$ in $L^{2}(\mathbb{R})$ does not depend on $t$.

${ }^{\star}$ This paper is a contribution to the Special Issue in honor of Anatol Kirillov and Tetsuji Miwa. The full collection is available at http://www.emis.de/journals/SIGMA/InfiniteAnalysis2013.html 
There are several interesting sets of initial data $\left\{u_{0}\right\}$ for which the Lax equation and the isospectral property of the family $\left\{L_{t}\right\}$ can be used to solve equation (1). Among these are the set of rapidly decreasing potentials $[11,35]$, which contains in particular the the class of classical reflectionless potentials $[6,11,22,35]$. The latter class gives rise to the soliton solutions of the KdV equation. Another family of initial data for which the Lax method "works" is that of the algebro-geometric potentials [7,39]. The algebro-geometric potentials are quasi-periodic in $x$. By passing to appropriate limits, one can solve the KdV equation for more general almost periodic initial data; see $[8,9,31,33]$ for more information concerning this matter.

In 1985, Lundina [34] introduced the family GR of generalized reflectionless Schrödinger potentials, which includes both the classical reflectionless potentials and (suitable translations of) the algebro-geometric potentials. In succeeding years, it was shown that (1) can be solved for various functions $u_{0}$ in GR (see, e.g., [14, 31, 35, 36, 37]; also [3, 13, 40]). In 2008, Kotani [27] proved that every element $u_{0} \in$ GR gives rise to a solution of (1), and indeed of the entire KdV hierarchy of evolution equations. He used the Sato-Segal-Wilson theory of the KdV hierarchy $[41,42]$. In fact, he was able to show that GR is contained in the Sato-Segal-Wilson family of potentials (see also [16] in this regard). In [22], it was shown that if a Sato-Segal-Wilson potential is suitably translated, then it lies in GR.

It is also well-known that one can determine soliton solutions and algebro-geometric solutions for various other nonlinear evolution equations and corresponding hierarchies, e.g., the SineGordon equation and the nonlinear Schrödinger equation. We will not dwell on this matter here, but will only note that the Camassa-Holm equation [5]

$$
\begin{aligned}
& y=2 f-\frac{1}{2} \frac{\partial^{2} f}{\partial x^{2}}, \\
& \frac{\partial y}{\partial t}=\frac{\partial y}{\partial x} f+2 y \frac{\partial f}{\partial x}
\end{aligned}
$$

is related to the Sturm-Liouville operator defined by

$$
-\varphi^{\prime \prime}+\varphi=\lambda y(x) \varphi
$$

in a fashion which is similar to the relation between the KdV equation (1) and the Schrödinger operator (2) [1, 2, 12, 44]. Motivated by this fact, we introduced in [23, 24] a hierarchy of evolution equations based on the general Sturm-Liouville spectral problem

$$
-\left(p \varphi^{\prime}\right)^{\prime}+q \varphi=\lambda y \varphi
$$

with positive weight $y$. This so-called Sturm-Liouville hierarchy includes both the KdV and the Camassa-Holm hierarchies as well as other evolution equations of interest (see Section 3). We also worked out a theory of algebro-geometric "potentials" $a=(p, q, y)$ for $(3)$ (see $[18,19])$, and showed how one can produce the solutions of the various equations in the Sturm-Liouville hierarchy which admit a given algebro-geometric potential as an initial condition [23].

Now, one can also define the concept of "generalized reflectionless Sturm-Liouville potentials" (see [20] and Section 2). However, there is as yet no analogue of the Sato-Segal-Wilson theory for the Sturm-Liouville potentials and the Sturm-Liouville hierarchy. For this and other reasons, it is of interest to construct solutions of the Sturm-Liouville hierarchy which have initial values in the class GRSL of generalized reflectionless Sturm-Liouville potentials but are not of algebro-geometric type. Our goal in this paper is to make a contribution in this direction. We will in fact consider certain limits of algebro-geometric potentials, and construct the corresponding solutions of the equations in the Sturm-Liouville hierarchy.

It is time to discuss in more detail the contents of the present paper. Let $p, q$ and $y$ be real valued functions of $x \in \mathbb{R}$ such that: $p, q$ and $y$ are all bounded uniformly continuous functions; 
$p \in C^{1}(\mathbb{R})$ and has a bounded uniformly continuous derivative $p^{\prime}(x) ; p$ and $y$ assume positive values and are bounded away from zero. The differential expression

$$
L_{a}=\frac{1}{y}\{-D p D+q\} \quad D=\frac{d}{d x}
$$

defines a self-adjoint operator on the weighted space $L^{2}(\mathbb{R}, y(x) d x)$. Suppose that this operator has spectrum $\Sigma=\left[\lambda_{0}, \lambda_{1}\right] \cup\left[\lambda_{2}, \lambda_{3}\right] \cup \cdots \cup\left[\lambda_{2 g}, \infty\right)$. The hypothesis that $a \in$ GRSL ensures that $a$ is of algebro-geometric type, in the sense that information about $a=(p, q, y)$ can be obtained by introducing the hyperelliptic Riemann surface $\mathcal{R}$ determined by the relation $w^{2}=$ $-\left(\lambda-\lambda_{0}\right)\left(\lambda-\lambda_{1}\right) \cdots\left(\lambda-\lambda_{2 g}\right)$, studying the motion of the zeroes of the diagonal Green's function by using the holomorphic differentials on $\mathcal{R}$ and the Abel map, etc. These matters are discussed in $[18,19,20]$, and part (but not all) of the discussion there is parallel to that found in previous literature on algebro-geometric solutions of hierarchies of evolution equations.

Let now suppose that the finite sequence $\lambda_{0}<\lambda_{1}<\cdots<\lambda_{2 g}$ is replaced by an infinite sequence $\lambda_{0}<\lambda_{1}<\cdots<\lambda_{2 g}<\cdots$ which tends to a limit $\lambda_{*} \leq \infty$. Set $\Sigma=\bigcup_{i=0}^{\infty}\left[\lambda_{2 i}, \lambda_{2 i+1}\right]$ if $\lambda_{*}=\infty$ and $\Sigma=\bigcup_{i=0}^{\infty}\left[\lambda_{2 i}, \lambda_{2 i+1}\right] \cup\left[\lambda_{*}, \infty\right)$ if $\lambda_{*}<\infty$. Let $a$ be a generalized reflectionless SturmLiouville potential which has spectrum $\Sigma$. It turns out that, under fairly general conditions on the sequence $\left\{\lambda_{i}\right\}$, such potentials exist, and moreover they serve as initial conditions giving rise to solutions of the Sturm-Liouville hierarchy. These facts were proved in [24] when $\lambda_{*}=\infty$, and our goal in the present paper is to prove them when $\lambda_{*}<\infty$. In particular we will obtain solutions of the Camassa-Holm hierarchy with generalized reflectionless initial data which, so far as we know, are new.

The proof of the existence of a generalized reflectionless Sturm-Liouville potential with spectrum $\Sigma$ proceeds by algebro-geometric approximation, as does the proof of the existence of a corresponding solution of the Sturm-Liouville hierarchy. This technique has been applied in the $\mathrm{KdV}$ case by several authors [4, 8, 9, 31, 33, 37, 45]. In the present case we find it convenient to deal with certain infinite products by using convergence factors similar to those of Weierstrass-Runge in the classical approximation theory of meromorphic functions [26]. So far as we know, this method has not been used when working out solutions of hierarchies of evolution equations by algebro-geometric approximation. We will see that it is quite convenient in the case of the Sturm-Liouville hierarchy.

The paper is organized as follows. In Section 2 we recall some basic facts concerning the algebro-geometric Sturm-Liouville potentials [18]. In Section 3 we review the construction of the Sturm-Liouville hierarchy of evolution equations and its solution for algebro-geometric initial data. We introduce the Weierstrass-Runge convergence factors [26] which, although unimportant in the algebro-geometric setting, seem necessary in order to manage the potentials and solutions which arise as limits when $g \rightarrow \infty$ and $\lambda_{2 g} \rightarrow \lambda_{*}<\infty$. Finally, in Section 4 we present the main results of this paper. Namely, we construct solutions of the Sturm-Liouville hierarchy whose initial data $a=(p, q, y)$ are of generalized reflectionless type, for which the corresponding operator has spectrum $\Sigma=\bigcup_{i=0}^{\infty}\left[\lambda_{2 i}, \lambda_{2 i+1}\right] \cup\left[\lambda_{*}, \infty\right)$ with $\lambda_{0}<\lambda_{1}<\cdots<\lambda_{2 g}<\cdots \rightarrow \lambda_{*}<\infty$.

\section{Some results on the Inverse Sturm-Liouville problem}

In this Section, we review some material concerning the study of the spectral theory of the Sturm-Liouville operator. For a detailed discussion concerning this topic, the reader is referred to $[18,19,20]$. 
Let $\mathcal{E}_{2}=\left\{b=(p, \mathcal{M}): \mathbb{R} \rightarrow \mathbb{R}^{2} \mid b\right.$ is uniformly continuous and $p(x) \geq \delta, \delta \leq \mathcal{M}(x) \leq \Delta$ for every $x \in \mathbb{R}\}$. Further, let $\mathcal{E}_{3}=\left\{a=(p, q, y): \mathbb{R} \rightarrow \mathbb{R}^{3} \mid a\right.$ is uniformly continuous and bounded, $p(x) \geq \delta, \delta \leq y(x) \leq \Delta$ for every $x \in \mathbb{R}\}$. Equip both $\mathcal{E}_{2}$ and $\mathcal{E}_{3}$ with the standard topology of uniform convergence on compact subsets of $\mathbb{R}$. Denote by $D$ the operator of differentiation with respect to $x$. If $a \in \mathcal{E}_{3}$ the Sturm-Liouville operator

$$
L_{a}: \mathcal{D} \rightarrow L^{2}(\mathbb{R}, y d x): \varphi \mapsto \frac{1}{y}(-D p D+q) \varphi
$$

is defined in its domain $\mathcal{D}=\left\{\varphi: \mathbb{R} \rightarrow \mathbb{R} \mid \varphi \in L^{2}(\mathbb{R}, y(x) d x)\right.$, $\varphi^{\prime}$ is absolutely continuous and $\left.\varphi^{\prime \prime} \in L^{2}(\mathbb{R}, y(x) d x)\right\}$. With a slight abuse of terminology, we refer to an element $a \in \mathcal{E}_{3}$ as a potential.

Now, $L_{a}$ admits a self-adjoint extension to all $L^{2}(\mathbb{R}, y(x) d x)$ (and we will continue to denote by $L_{a}$ this extension as well), hence its spectrum $\Sigma_{a}$ is contained in $\mathbb{R}$, is bounded below and unbounded above, and its resolvent set $R_{a}=\mathbb{R} \backslash \Sigma_{a}$ is at most a countable union (possibly unbounded) of disjoint open intervals. Notice that the operators we are dealing with include the Schrödinger operator (obtained with $a=(1, q, 1)$ ) and the so-called acoustic operator (when $a=(1,1, y))$.

As already remarked in the Introduction, the spectral theory of the Sturm-Liouville operator is important both for its intrinsic value, and for the connection existing between this kind of operator and the solutions of some important evolution equations such as the KdV equation, the Camassa-Holm equation, and other recently discovered evolution equations.

So, we discuss some facts concerning the spectral theory of the Sturm-Liouville operator. It has turned out that it is convenient to attack this problem by using instruments of the theory of nonautonomous dynamical systems. To each $a \in \mathcal{E}_{3}$ and the corresponding operator $L_{a}$, one associates the eigenvalue equation

$$
E_{a}(\varphi, \lambda):=-\left(p \varphi^{\prime}\right)^{\prime}+q \varphi=\lambda y \varphi, \quad \lambda \in \mathbb{C} .
$$

This equation can be expressed as follows

$$
X^{\prime}=A(x, \lambda) X=\left(\begin{array}{cc}
0 & 1 / p(x) \\
q(x)-\lambda y(x) & 0
\end{array}\right) X, \quad X=\left(\begin{array}{c}
\varphi(x) \\
p(x) \varphi^{\prime}(x)
\end{array}\right) .
$$

Now, let $A: \mathcal{E}_{3} \times \mathbb{C} \rightarrow \mathbb{M}(2, \mathbb{C}):(a, \lambda) \mapsto A(0, \lambda)$. Denote by $\left\{\tau_{s}\right\}$ the Bebutov (or translation) flow on $\mathcal{E}_{3}$, i.e., if $a(\cdot) \in \mathcal{E}_{3}$, we define $\tau_{s}(a)=a(s+\cdot) \in \mathcal{E}_{3}$. Fix $a_{0} \in \mathcal{E}_{3}$, and let $\mathcal{A}=$ $\operatorname{cls}\left\{\tau_{s}\left(a_{0}\right) \mid s \in \mathbb{R}\right\}$ (cls denotes the topological closure). One calls $\mathcal{A}$ the Hull of $a_{0}$ and writes $\mathcal{A}=\operatorname{Hull}\left(a_{0}\right)$. Since $a_{0}$ is uniformly continuous, then $\mathcal{A}$ is a compact subset of $\mathcal{E}_{3}$. Moreover $\mathcal{A}$ is also invariant, in the sense that $\tau_{s}(\mathcal{A})=\mathcal{A}$ for every $s \in \mathbb{R}$. This construction (said to be of Bebutov type) allows one to use the instruments of topological dynamics to study the spectral theory of the operators. We will not pause to show how this takes place, however, we will briefly introduce some objects which will be important in the following pages.

It is clear that the construction we made above leaves us with a family of linear systems, namely

$$
\left(\begin{array}{c}
\varphi \\
p \varphi^{\prime}
\end{array}\right)^{\prime}=A\left(\tau_{x}(a), \lambda\right)\left(\begin{array}{c}
\varphi \\
p \varphi^{\prime}
\end{array}\right), \quad a \in \mathcal{A}, \quad \lambda \in \mathbb{C} .
$$

The fundamental tool to study the systems (4) is the concept of exponential dichotomy. For $a \in \mathcal{A}$ and $\lambda \in \mathbb{C}$, let $\Phi_{a}(x)$ be the fundamental matrix solution of the corresponding equation in (4): 
Definition 1. The family (4) is said to have an exponential dichotomy over $\mathcal{A}$ if there are positive constants $\eta, \rho$, together with a continuous, projection valued function $P: \mathcal{A} \rightarrow \mathbb{M}_{2}(\mathbb{C})$ such that the following estimates holds:

(i) $\left|\Phi_{a}(x) P(a) \Phi_{a}(s)^{-1}\right| \leq \eta e^{-\rho(x-s)}, \quad x \geq s$,

(ii) $\left|\Phi_{a}(x)(I-P(a)) \Phi_{a}(s)^{-1}\right| \leq \eta e^{\rho(x-s)}, \quad x \leq s$.

One has the following fundamental result (see $[15,17])$.

Theorem 1. Let $\mathcal{A}$ be obtained by a Bebutov type construction as above. Consider the family (4). If $a \in \mathcal{A}$ has dense orbit, then the spectrum $\Sigma_{a}$ of the operator $L_{a}$ equals the set

$\Sigma_{\text {ed }}:=\{\lambda \in \mathbb{C} \mid$ the family (4) does not admit an exponential dichotomy over $\mathcal{A}\}$.

It is known that, if $\Im \lambda \neq 0$, then the family (4) admits an exponential dichotomy over $\mathcal{A}$ (and indeed $\Sigma_{a} \subset \mathbb{R}$ ). Moreover, if $a \in \mathcal{E}_{3}$ and $\mathcal{A}=\operatorname{Hull}(a)$ then the spectrum of $L_{a}$ and that of all the operators $L_{\tau_{x}(a)}$ coincide, i.e., $\Sigma_{a}=\Sigma_{\tau_{x}(a)}=\Sigma_{\text {ed }}$ for every $x \in \mathbb{R}[10]$.

Now, let $a \in \mathcal{E}_{3}$ and let us fix the Dirichlet boundary condition $\varphi(0)=0$. There are welldefined unbounded self-adjoint operators $L_{a}^{ \pm}$which are defined in $L^{2}\left(\mathbb{R}^{ \pm}, y(x) d x\right)$ and which are determined via the relation

$$
L_{a}(\varphi)=\frac{1}{y}\left[-\left(p \varphi^{\prime}\right)^{\prime}+q \varphi\right]
$$

and the Dirichlet boundary condition at $x=0$. If $\Im \lambda \neq 0$, we define the Weyl $m$-functions $m_{ \pm}(a, \lambda)$ as those complex numbers which parametrize $\operatorname{Ker} P(a)$ and $\operatorname{Im} P(a)$, as follows:

$$
\operatorname{Im} P(a)=\operatorname{Span}\left(\begin{array}{c}
1 \\
m_{+}(a, \lambda)
\end{array}\right), \quad \operatorname{Ker} P(a)=\operatorname{Span}\left(\begin{array}{c}
1 \\
m_{-}(a, \lambda)
\end{array}\right) .
$$

Note that, since $a \in \mathcal{A}$ and $\operatorname{det} \Phi_{a}(x)=1$ for every $x \in \mathbb{R}$, both $\operatorname{Ker} P(a)$ and $\operatorname{Im} P(a)$ are complex lines in $\mathbb{C}^{2}$. Since $\tau_{x}(a) \in \mathcal{A}$ for every $x \in \mathbb{R}$, the functions $m_{ \pm}\left(\tau_{x}(a), \lambda\right):=m_{ \pm}(x, \lambda)$ are well defined. They satisfy the Riccati equation

$$
m^{\prime}+\frac{1}{p} m^{2}=q-\lambda y, \quad \Im \lambda \neq 0 .
$$

Next, let $a=(p, q, y) \in \mathcal{E}_{3}$ be a Sturm-Liouville potential. Consider the (unbounded, selfadjoint) operator $L_{a}=\frac{1}{y}(-D p D+q)$ on $L^{2}(\mathbb{R}, y(x) d x)$ with domain $\mathcal{D}$. We will define the Green's function for the operator $L_{a}$. The Green's function $\mathcal{G}_{a}(x, s, \lambda)$ is the kernel of the resolvent operator $\left(L_{a}-\lambda I\right)^{-1}$ acting on $L^{2}(\mathbb{R}, y(x) d x)(\Im \lambda \neq 0)$. This means that, if one considers the nonhomogeneous equation $-\left(p \psi^{\prime}\right)^{\prime}+q \psi=\lambda y \psi+y f$, where $f \in L^{2}(\mathbb{R}, y(x) d x)$ and if $\Im \lambda \neq 0$, one has

$$
\psi(x)=\int_{\mathbb{R}} \mathcal{G}_{a}(x, s, \lambda) f(s) d s .
$$

If $a \in \mathcal{A}$, the Weyl $m$-functions $m_{ \pm}(x, \lambda)$ and the diagonal Green's function $\mathcal{G}_{a}(x, \lambda):=\mathcal{G}_{a}(x, x, \lambda)$ are connected by the fundamental relation

$$
\mathcal{G}_{a}(x, \lambda)=\frac{y(x)}{m_{-}(x, \lambda)-m_{+}(x, \lambda)}, \quad \Im \lambda \neq 0 .
$$

The above formula implies that

$$
\mathcal{G}_{a}(x, \lambda)=\mathcal{G}_{\tau_{x}(a)}(0,0, \lambda), \quad x \in \mathbb{R}, \quad \Im \lambda \neq 0 .
$$


It is known that, for every $x \in \mathbb{R}$, the non-tangential limit

$$
\mathcal{G}_{a}(x, \eta):=\lim _{\varepsilon \rightarrow 0} \mathcal{G}_{a}(x, \eta+i \varepsilon)
$$

exists for a.a. $\eta \in \mathbb{R}$. In general, it is the behavior of the function $\mathcal{G}_{a}(x, \lambda)$ which provides a division of $\mathcal{E}_{3}$ into subsets which we will call spectral classes. Here, we mention only two of the most important spectral classes which exist, namely the algebro-geometric and the reflectionless spectral classes.

Definition 2. (I) A potential $a \in \mathcal{E}_{3}$ belongs to the algebro-geometric spectral class (briefly, is algebro-geometric) if it enjoys the following properties:

1) the spectrum $\Sigma_{a}$ of the operator $L_{a}$ is a finite union of disjoint compact intervals, plus a half-line:

$$
\Sigma_{a}=\left[\lambda_{0}, \lambda_{1}\right] \cup\left[\lambda_{2}, \lambda_{3}\right] \cup \cdots \cup\left[\lambda_{2 g}, \infty\right) .
$$

2) for every $x \in \mathbb{R}$, one has $\Re \mathcal{G}_{a}(x, \eta)=0$, for a.a. $\eta \in \Sigma_{a}$.

(II) A potential $a \in \mathcal{E}_{3}$ belongs to the reflectionless spectral class (or is simply reflectionless) if:

1) the spectrum $\Sigma_{a}$ has locally positive Lebesgue measure, in the sense that if $\eta \in \Sigma_{a}$ and if $I \subset \mathbb{R}$ is an open interval with $\eta \in I$, then $I \cap \Sigma_{a}$ has positive Lebesgue measure;

2) for every $x \in \mathbb{R}$, there holds $\Re \mathcal{G}_{a}(x, \eta)=0$ for a.a. $\eta \in \Sigma_{a}$.

(III) A family of potentials $\{a\}_{a \in \mathcal{F}}$ lies in the isospectral class of $a_{0} \in \mathcal{E}_{3}$ if, for every $a \in \mathcal{F}$, the spectrum of the operator $L_{a}$ equals the spectrum of the operator $L_{a_{0}}$.

It would perhaps be more appropriate to speak of generalized reflectionless instead of reflectionless potentials, but we prefer the simpler terminology. Our definition follows Craig [6].

The condition (2) in the above definitions has some fundamental consequences: indeed, it turns out that, for every $x \in \mathbb{R}$, both the maps $\lambda \mapsto m_{ \pm}(x, \lambda)(\Im \lambda \neq 0)$ extend holomorphically through every open set contained in the spectrum $\Sigma_{a}$. If $h_{ \pm}(x, \lambda)$ denote these extensions, we have

$$
h_{+}(x, \lambda)=\left\{\begin{array}{ll}
m_{+}(x, \lambda), & \Im \lambda>0, \\
m_{-}(x, \lambda), & \Im \lambda<0
\end{array} \quad \text { and } \quad h_{-}(x, \lambda)= \begin{cases}m_{-}(x, \lambda), & \Im \lambda>0, \\
m_{+}(x, \lambda), & \Im \lambda<0 .\end{cases}\right.
$$

Other fundamental properties of an algebro-geometric potential $a \in \mathcal{E}_{3}$ can be summarized as follows:

1. The spectrum $\Sigma_{a}$ does not contain any isolated eigenvalues.

2. The functions $m_{ \pm}(a, \cdot)$ extend meromorphically through the resolvent set $R_{a}=\mathbb{R} \backslash \Sigma_{a}$. Let $I_{j}=\left[\lambda_{2 j-1}, \lambda_{2 j}\right]$ be the closure of an interval of the resolvent set $(j=1, \ldots, g)$. It turns out that in $I_{j}$ there exists exactly one point $P_{j}(a)$ with the following property: either $m_{+}\left(a, P_{j}(a)+i \varepsilon\right)$ or $m_{-}\left(a, P_{j}(a)+i \varepsilon\right)$ has a simple pole as $\varepsilon \rightarrow 0$. The points $P_{j}(a)$ correspond to the isolated eigenvalues of the half-line restricted operators $L_{a}^{ \pm}$with the boundary condition $\varphi(0)=0$.

3. The properties (1) and (2) hold also for every potential $\tau_{x}(a)(x \in \mathbb{R})$, hence we are left with the functions $m_{ \pm}(x, \lambda)$ which extend meromorphically through the resolvent set, and with the poles $P_{j}(x):=P_{j}\left(\tau_{x}(a)\right)$. 
The observations made so far have an important consequence. Let $a \in \mathcal{E}_{3}$ be algebrogeometric, with spectrum $\Sigma_{a}=\left[\lambda_{0}, \lambda_{1}\right] \cup\left[\lambda_{2}, \lambda_{3}\right], \cup \cdots \cup\left[\lambda_{2 g}, \infty\right)$. To such a potential $a$ there are associated the poles $P_{1}(x), \ldots, P_{g}(x)$ described in the above lines. Let us assume from now on $\lambda_{0}>0$ - though of course one can define and discuss algebro-geometric Sturm-Liouville potentials when $\lambda_{0}<0$ (see [17]). Let $\mathcal{R}$ be the Riemann surface of the relation

$$
w^{2}=-\left(\lambda-\lambda_{0}\right)\left(\lambda-\lambda_{1}\right) \cdots\left(\lambda-\lambda_{2 g}\right) .
$$

Then $\mathcal{R}$ is a torus with $g$ holes which correspond to the spectral gaps $I_{j}=\left[\lambda_{2 j-1}, \lambda_{2 j}\right](j=$ $1, \ldots, g)$. It is a standard method now to consider the projection $\pi: \mathcal{R} \rightarrow \mathbb{C}_{\infty}$ (where $\mathbb{C}_{\infty}$ is the Riemann sphere). The projection $\pi$ is $2-1$, except at the points $\lambda_{0}, \lambda_{1}, \ldots, \lambda_{2 g}, \infty$ where it is 1-1. We call these points the ramification points of $\mathcal{R}$. If $\lambda \in \mathbb{C}_{\infty}$ is not a ramification point, then there are two points $P_{+}$and $P_{-}$on $\mathcal{R}$ such that $\pi\left(P_{ \pm}\right)=\lambda$. Define a function $k(P)$ on $\mathcal{R}$ by setting $k^{2}(\lambda)=-\left(\lambda-\lambda_{0}\right) \cdots\left(\lambda-\lambda_{2 g}\right)$, then letting $k\left(0^{ \pm}\right)$be the positive/negative square root of $\lambda_{0} \lambda_{1} \cdots \lambda_{2 g}$, and then extending via analytic continuation. The result is a well-defined function $P \mapsto k(P)$ on $\mathcal{R}$.

Further, let us define $c_{j}=\pi^{-1}\left(\left[\lambda_{2 j-1}, \lambda_{2 j}\right]\right)(j=1, \ldots, g)$. Then $c_{j}$ are circles corresponding to the inner boundary of $\mathcal{R}$. For a point $P_{j} \in c_{j}$, its projection $\pi\left(P_{j}\right)$ lies in $I_{j}$. We agree that $k\left(P_{j}\right)$ is positive or negative according to the position of $P_{j}$ on the circle $c_{j}$. In particular, if we express $P_{j}$ as

$$
P_{j}=\left(\lambda_{2 j-1}-\lambda_{2 j}\right) \sin ^{2} \frac{\theta_{j}}{2}+\lambda_{2 j}, \quad \theta_{j} \in[0,2 \pi],
$$

then $k\left(P_{j}\right)>0$ if $\theta_{j} \in(0, \pi)$, while $k\left(P_{j}\right)<0$ if $\theta_{j} \in(\pi, 2 \pi)$. This convention will exclude every possible misunderstanding in the future. Also, we will often commit an abuse of notation in denoting by $P_{j}$ both the point in $c_{j}$ and its projection in $I_{j}$.

The setting we have introduced clarifies the reason of the name algebro-geometric. Indeed, the spectral properties of the operator $L_{a}$ can now be described by moving to the Riemann surface $\mathcal{R}$. We briefly discuss this (see $[18,19]$ for details).

Let $a=(p, q, y) \in \mathcal{E}_{3}$ be an algebro-geometric potential. Hence its spectrum $\Sigma_{a}=\left[\lambda_{0}, \lambda_{1}\right] \cup$ $\cdots \cup\left[\lambda_{2 g, \infty}\right)$ is given, the Weyl $m$-functions $m_{ \pm}(x, \lambda)$ behave properly, together with their poles $P_{1}(x), \ldots, P_{g}(x)$ (or, equivalently, the isolated eigenvalues of the half-line restricted operators $L_{\tau_{x}(a)}^{ \pm}$. If we concentrate further on the behavior of the Weyl $m$-functions, we can argue that, for every $x \in \mathbb{R}$, one can define a single meromorphic function $M: \mathcal{R} \rightarrow \mathbb{C}_{\infty}$ by setting, as before, $M\left(x, 0^{ \pm}\right)=m_{ \pm}(x, 0)$, and then using analytic continuation on $\mathcal{R}$. Again, for every fixed $x \in \mathbb{R}$, one can define $m_{+}(x, P)=M(x, P)$ and $m_{-}(x, P)=M(x, \sigma(P))$, where $\sigma$ is the hyperelliptic involution (the map which changes the sheets). Actually, all these maps are jointly continuous when viewed as defined on $\mathbb{R} \times(\mathbb{C} \backslash \mathbb{R})$. Now, expanding $M$ at $\infty$, we obtain

$$
\begin{aligned}
& M(x, P)=m_{+}(x, P)=\frac{Q(x, \lambda)+\sqrt{p(x) y(x)} k(P)}{H(x, \lambda)}, \\
& m_{-}(x, P)=\frac{Q(x, \lambda)-\sqrt{p(x) y(x)} k(P)}{H(x, \lambda)},
\end{aligned}
$$

where $\lambda=\pi(P), Q(x, \lambda)$ is a polynomial in $\lambda$ of degree $g$, and

$$
H(x, \lambda)=\prod_{i=1}^{g}\left(\lambda-\pi\left(P_{i}(x)\right)\right) .
$$

Moreover, it turns out that

$$
Q(x, \lambda)=\frac{p(x)}{2} \sqrt{p(x) y(x)}\left(\frac{1}{\sqrt{p(x) y(x)}} H(x, \lambda)\right)_{x} .
$$


Here and throughout all the paper the subscripts $(\cdot)_{s}$ denote the (partial) derivative with respect to a variable $s$.

Recall that $\lambda_{0}>0$. Set $\mathcal{M}(x)=m_{-}(x, 0)-m_{+}(x, 0)$. Using the Riccati equation (5), one can show that

$$
P_{i, x}(x)=\frac{-\mathcal{M}(x) k\left(P_{i}(x)\right) \prod_{i=1}^{g} P_{i}(x)}{p(x) k\left(0^{+}\right) \prod_{j \neq i}\left(P_{j}(x)-P_{i}(x)\right)}, \quad i=1, \ldots, g .
$$

The equations in (6) provide a system of $g$ ODE's. The induced flow is intended to take place on $\mathbb{R}$, hence we must take care of the value $k(P)$, according to the observations we made above. However, it is possible to pass to polar coordinates and write down a system for the angular coordinate $\theta_{i}(x)$ of each pole $P_{i}(x)$, avoiding any type of confusion. Clearly, given an initial condition $P_{1}(0), \ldots, P_{g}(0)$, the system (6) admits a unique, globally defined solution, which we call the pole motion. Once the pole motion is determined, we can write down the so-called trace formulas for the potential $a=(p, q, y)$, namely

$$
\begin{gathered}
y(x)=\frac{\mathcal{M}^{2}(x) \prod_{i=1}^{g} P_{i}^{2}(x)}{4 p(x) k^{2}\left(0^{+}\right)} \\
q(x)=y(x)\left(\lambda_{0}+\sum_{i=1}^{g} \lambda_{2 i-1}+\lambda_{2 i}-2 P_{i}(x)\right)+\tilde{q}(x),
\end{gathered}
$$

where

$$
\tilde{q}(x)=-\left(\frac{(p(x) y(x))_{x}}{4 y(x)}\right)_{x}+\left(\frac{(p(x) y(x))_{x}}{4 y(x)}\right)^{2} .
$$

It is a recent discovery (to appear in a forthcoming paper [25]) that the function $\tilde{q}(x)$ plays a crucial role in a development of a theory of Gel'fand-Levitan-Marchenko type for the SturmLiouville operator.

We finish this section by establishing the way to reconstruct an algebro-geometric potential $a=(p, q, y)$ from some given spectral data. Let us fix $(p, \mathcal{M}) \in \mathcal{E}_{2}$. Choose the spectral parameters, namely the ordered set

$$
\left\{0<\lambda_{0}<\lambda_{1} \leq P_{1}(0) \leq \lambda_{2}<\lambda_{3} \leq P_{2}(0) \leq \lambda_{4}<\cdots<\lambda_{2 g-1} \leq P_{g}(0) \leq \lambda_{2 g}\right\} .
$$

Let $P_{1}(x), \ldots, P_{g}(x)$ be the solution of the system (6) with initial condition $P_{1}(0), \ldots, P_{g}(0)$. Finally, define $y(x)$ and $q(x)$ as to satisfy the relations (7) and (8). The triple $a=(p, q, y) \in \mathcal{E}_{3}$ thus defined is an algebro-geometric potential whose spectrum $\Sigma_{a}$ is given by $\Sigma_{a}=\left[\lambda_{0}, \lambda_{1}\right] \cup$ $\cdots \cup\left[\lambda_{2 g}, \infty\right)$.

\section{The Sturm-Liouville hierarchy of evolution equations revisited}

The Sturm-Liouville hierarchy of evolution equations has been introduced and studied in detail in $[23,24]$. In those papers, we determined certain solutions of the hierarchy: namely, the algebro-geometric solutions and some types of solutions whose initial data are related to particular classes of reflectionless potentials. In this paper, we extend the family of solutions we are able to describe by enlarging the class of admissible initial conditions. The initial conditions we 
introduce in the following lie in the reflectionless spectral class as well. They are of a type which generalizes the Schroedinger potentials considered in [4, 8, 9, 32]. Namely, these initial SturmLiouville data have spectrum which clusters at finite points of $\mathbb{R}$. To include these potentials in the discussion, we will need to slightly modify the structure of the hierarchy. At first sight, some quantities we will introduce soon will not be significant, but they will be fundamental when a limit procedure will be carried out.

But let us start by describing what we mean by Sturm-Liouville hierarchy of evolution equations. For convenience, we will first choose the initial data, then define the evolution equations which will be solved. Let $(p, \mathcal{M}) \in \mathcal{E}_{2}$ and choose arbitrarily the spectral parameters, i.e., the set

$$
\Lambda_{g}=\left\{0<\lambda_{0}<\lambda_{1} \leq P_{1}(0) \leq \lambda_{2}<\lambda_{3} \leq P_{2}(0) \leq \lambda_{4}<\cdots<\lambda_{2 g-1} \leq P_{g}(0) \leq \lambda_{2 g}\right\} .
$$

Then an algebro-geometric potential $a=(p, q, y) \in \mathcal{E}_{3}$, and the associated Sturm-Liouville operator $L_{a}$ with prescribed spectrum $\Sigma_{g}=\left[\lambda_{0}, \lambda_{1}\right] \cup \cdots \cup\left[\lambda_{2 g}, \infty\right)$ can be determined.

For $\lambda \in \mathbb{C}$, let us set

$$
E_{n}(\lambda)=\exp \left(\lambda+\frac{\lambda^{2}}{2}+\cdots+\frac{\lambda^{n}}{n}\right)
$$

Next, fix a point $\lambda_{*} \in \mathbb{R}^{+} \backslash \Lambda_{g}$. Define a function

$$
U_{g}(x, \lambda)=\frac{-2 p(x) k\left(0^{+}\right)}{\mathcal{M}(x) \prod_{i=1}^{g} P_{i}(x)} \prod_{i=1}^{g} \frac{\lambda-P_{i}(x)}{\lambda_{*}-\lambda} E_{i}\left(\frac{\lambda_{*}-\lambda_{2 i}}{\lambda_{*}-\lambda}\right) .
$$

Clearly $U_{g}(x, \cdot)$ is defined in the punctured complex plane $\mathbb{C} \backslash\left\{\lambda_{*}\right\}$ and has an essential singularity at $\lambda=\lambda_{*}$. Further, define

$$
\tilde{k}_{g}^{2}(\lambda)=\left(\lambda_{0}-\lambda\right) \prod_{i=1}^{g}\left(\frac{\lambda-\lambda_{2 i}}{\lambda-\lambda_{*}}\right)\left(\frac{\lambda-\lambda_{2 i-1}}{\lambda-\lambda_{*}}\right) E_{i}^{2}\left(\frac{\lambda_{*}-\lambda_{2 i}}{\lambda_{*}-\lambda}\right) .
$$

It is clear that the function $\tilde{k}_{g}^{2}(\lambda)$ is strictly related to the function $k(P)$ defined in the previous section.

Let us observe that in $[23,24]$, we introduced the analogues of the functions $\tilde{k}_{g}$ and $U_{g}$ in which the terms $E_{i}$ and $\frac{1}{\lambda_{*}-\lambda}$ are not present. They are introduced here with an eye to the limit procedure which will be carried out in Section 4. We state without giving all the details that the theory of $[23,24]$ can be developed beginning with $U_{g}$ and $\tilde{k}_{g}$ as given in (9) and (10), as well as the simpler forms of $U_{g}$ and $\tilde{k}_{g}$ in $[23,24]$ (which, to repeat, do not have the functions $E_{i}$ and $\frac{1}{\lambda_{*}-\lambda}$ ). We proceed to outline this (modified) theory.

Choose an integer $0 \leq k \leq g-1$, and define two additional functions $T_{g}(x, \lambda)$ and $V_{g}(x, \lambda)$ in such a way that

$$
T_{g}(x, \lambda)=\frac{p(x)}{2 \lambda^{k}}\left(\frac{U_{g}(x, \lambda)}{p(x)}\right)_{x}
$$

and

$$
T_{g, x}(x, \lambda)+\frac{1}{\lambda^{k} p(x)}(q(x)-\lambda y(x))\left(V_{g}(x, \lambda)-U_{g}(x, \lambda)\right)=0 .
$$

Set

$$
B_{g}=\left(\begin{array}{cc}
-T_{g} & \lambda^{-k} U_{g} / p \\
\lambda^{-k}(q-\lambda y) V_{g} & T_{g}
\end{array}\right)
$$


and, as usual

$$
A=\left(\begin{array}{cc}
0 & 1 / p \\
q-\lambda y & 0
\end{array}\right)
$$

It can be shown (see $[23,24])$ that the so-called stationary zero-curvature relation holds, namely

$$
-B_{g, x}+\left[A, B_{g}\right]=0,
$$

where $\left[A, B_{g}\right]=A B_{g}-B_{g} A$ is the commutator of $A$ and $B_{g}$. Moreover, there holds

$$
\frac{d}{d x} \operatorname{det} B_{g}=0,
$$

which translates into the fundamental relation

$$
\frac{p^{2}}{4}\left[\left(\frac{U_{g}}{p}\right)_{x}\right]^{2}+\frac{1}{p}(q-\lambda y) U_{g} V_{g}=\tilde{k}_{g}^{2}(\lambda) .
$$

Actually, more can be proved. We state the following result; see [23, 24].

Theorem 2. If a potential $a=(p, q, y) \in \mathcal{E}_{3}$ is algebro-geometric with spectral parameters

$$
\Lambda_{g}=\left\{0<\lambda_{0}<\lambda_{1} \leq P_{1}(0) \leq \lambda_{2}<\lambda_{3} \leq P_{2}(0) \leq \lambda_{4}<\cdots<\lambda_{2 g-1} \leq P_{g}(0) \leq \lambda_{2 g}\right\},
$$

then there exist functions $U_{g}(x, \lambda), \tilde{k}_{g}^{2}(\lambda), T_{g}(x, \lambda)$ and $V_{g}(x, \lambda)$ as in the relations (9)-(12) respectively such that the zero curvature relation $-B_{g, x}+\left[A, B_{g}\right]=0$ holds, together with the relation (13).

Conversely, let $a \in \mathcal{E}_{3}$, and suppose that the left endpoint of the spectrum of $L_{a}$ equals $\lambda_{0}>0$. Let $\mathcal{M}(x)=m_{-}(x, 0)-m_{+}(x, 0)$. Suppose that one can determine $U_{g}(x, \lambda)$ together with the corresponding quantities $\tilde{k}_{g}^{2}(\lambda), T_{g}(x, \lambda)$ and $V_{g}(x, \lambda)$ so that relations $(9)-(12)$ hold, and so that the zero curvature relation $-B_{g, x}+\left[A, B_{g}\right]=0$ and (13) are valid. Then a is of algebro-geometric type.

We are now ready to introduce the Sturm-Liouville hierarchy of evolution equations. It is here that the integer $k$ becomes significant. We let a parameter $t$ enter into play. One obtains functions $a(t, x)=(p(t, x), q(t, x), y(t, x))$ and $\mathcal{M}(t)$ producing the poles $P_{1}(t, x), \ldots, P_{g}(t, x)$, and functions as in (9)-(12) where the variable $t$ is present. For instance, we will have a function

$$
U_{g}(t, x, \lambda)=\frac{-2 p(t, x) k\left(0^{+}\right)}{\mathcal{M}(t, x) \prod_{i=1}^{g} P_{i}(t, x)} \prod_{i=1}^{g} \frac{\lambda-P_{i}(t, x)}{\lambda_{*}-\lambda} E_{i}\left(\frac{\lambda_{*}-\lambda_{2 i}}{\lambda_{*}-\lambda}\right),
$$

and so on. In this way one has matrices $B_{g}(t, x, \lambda)$ and $A(t, x, \lambda)$. If we force $a(t, \cdot)$ to lie in the algebro-geometric isospectral class of $a(0, \cdot)$, then for every $t \in \mathbb{R}$ one has the stationary zero-curvature relation

$$
-B_{g, x}(t, x, \lambda)+\left[A(t, x, \lambda), B_{g}(t, x, \lambda)\right]=0
$$

together with the relation (13), which now expresses the invariance with respect to $t$ of its r.h.s. member as well.

However, we must still determine the time evolution of the functions we have introduced. We do this as follows: fix an integer $r$ such that $0 \leq k \leq r<g$. Introduce a new matrix $B_{r}(t, x, \lambda)$ of the form

$$
B_{r}(t, x, \lambda)=\left(\begin{array}{cc}
-T_{r}(t, x, \lambda) & \lambda^{-k} \frac{U_{r}(t, x, \lambda)}{p(t, x)} \\
\lambda^{-k}(q(t, x)-\lambda y(t, x)) V_{r}(t, x, \lambda) & T_{r}(t, x, \lambda)
\end{array}\right),
$$


where $U_{r}$ is a polynomial of degree $r$ in $\lambda$ (whose coefficients depend on $t$ and $x$ ), and $T_{r}(t, x, \lambda)$ and $V_{r}(t, x, \lambda)$ are defined via the relations

$$
\begin{aligned}
& \left(\frac{1}{p}\right)_{t}-\lambda^{-k}\left(\frac{U_{r}}{p}\right)_{x}+\frac{2}{p} T_{r}=0, \\
& T_{r, x}+\frac{\lambda^{-k}}{p}(q-\lambda y)\left(V_{r}-U_{r}\right)=0 .
\end{aligned}
$$

We pose the following basic question [23, 24]

Question 1. Can $U_{g}(t, x, \lambda)$ and $U_{r}(t, x, \lambda)$ be chosen in such a way that

$$
\begin{aligned}
& -B_{g, x}+\left[A, B_{g}\right]=0, \\
& A_{t}-B_{r, x}+\left[A, B_{r}\right]=0, \\
& \frac{d}{d x} \operatorname{det} B_{g}=0, \quad \text { and (13) holds }
\end{aligned}
$$

for all $(t, x) \in \mathbb{R}^{2}$ and all $\lambda \neq \lambda_{*}$ ?

It is understood that $B_{g}$ satisfies the conditions discussed above, and that $B_{r}$ satisfies certain auxiliary conditions which will be discussed in due course (see [23]).

The second equation in the system (16) is called the zero-curvature relation, and the system (16) determines the Sturm-Liouville evolution equation of order $r$ in a way which we will explain in a few lines. Before doing so, we point out that the first and the third equations in (16) force the potentials $a(t, x)=(p(t, x), q(t, x), y(t, x))$ to lie in the same isospectral class of $a(x):=a(0, x)=(p(0, x), q(0, x), y(0, x))$, i.e., if we fix $a(0, x)$ as initial data, the whole motion $t \mapsto a(t, x)$ will take place in its isospectral class. To change the initial data means to change the matrix $B_{g}(0, x, \lambda)$ and the r.h.s. of $(13)$ ! This change will have an effect on $B_{r}$ as well because of the zero-curvature relation!

Before answering the above question, we explain how it translates into a single evolution equation. Let us set $\tilde{U}_{r}=U_{r} / p$. It turns out that $\tilde{U}_{r}$ must satisfy the relation

$$
\begin{aligned}
& 2 \lambda^{k}(q-\lambda y)_{t}+\frac{p_{t}}{p}(q-\lambda y)+\left(p\left(\frac{p_{t}}{p}\right)_{x}\right)_{x} \\
& \quad=2(p(q-\lambda y))_{x} \tilde{U}_{r}+4 p(q-\lambda y) \tilde{U}_{r, x}-\left(p\left(p \tilde{U}_{r, x}\right)_{x}\right)_{x} .
\end{aligned}
$$

We make the fundamental ansatz that $\tilde{U}_{r}$ (and hence $U_{r}$ ) be a polynomial of degree $r$ in $\lambda$, i.e.,

$$
\tilde{U}_{r}(t, x, \lambda)=\sum_{j=0}^{r} f_{j}(t, x) \lambda^{j} .
$$

If this is true, then the relation (17) provides $r+2$ recursion relations: it can be shown that, once we fix a pair $(p(t, x), \mathcal{M}(t, x)) \in \mathcal{E}_{2}$, then one of the coefficients of $\tilde{U}_{r}$ is determined without using these recursion relations, hence $r$ of the recursion relations will be used to find all the coefficients $f_{j}(t, x)$. There remain 2 relations. These 2 relations are compatibility conditions for (16), and translate into 2 evolution equations, one for the function $q(t, x)$ and the other for the function $y(t, x)$. It is this pair of equations which we call the Sturm-Liouville hierarchy of evolution equations. In more detail, these 2 equations correspond to the formulas in (17) when we try to determine the coefficients of $\lambda^{k}$ and $\lambda^{k+1}$. They give rise to relations of the type (here $\left.f_{-1}=f_{r+1}=0\right)$

$$
\begin{aligned}
& q_{t}=\mathcal{Q}_{r}\left(t, x, f_{k-1}, f_{k}, q, q_{x}, q_{x x}, \ldots, y, y_{x}, y_{x x}, p, p_{x}, p_{x x}, \ldots\right), \\
& y_{t}=\mathcal{Y}_{r}\left(t, x, f_{k}, f_{k+1}, q, q_{x}, q_{x x}, \ldots, y, y_{x}, y_{x x}, p, p_{x}, p_{x x}, \ldots\right) .
\end{aligned}
$$

Question 1 can now be formulated in the following convenient form 
Question 2. Does there exist a polynomial $U_{r}(t, x, \lambda)$ of degree $r$ in $\lambda$ (and satisfying certain auxiliary conditions),

$$
U_{r}(t, x, \lambda)=\sum_{j=0}^{r} p(t, x) f_{j}(t, x) \lambda^{j}
$$

such that, if $\tilde{U}_{r}=U_{r} / p$ and $T_{r}(t, x, \lambda)$ and $V_{r}(t, x, \lambda)$ are defined as in (14) and (15), then the system (16) admits a unique solution, once the triple $a(0, x)=(p(0, x), q(0, x), y(0, x))$ is a given algebro-geometric potential?

Before giving an answer to Question 2, we give concrete examples of some evolution equations which can be obtained with this procedure. Let $k=0$, and fix $p(t, x)=y(t, x)=1$. Then $\tilde{U}_{r}=U_{r}$ and (17) reads

$$
2 q_{t}=2 q_{x} U_{r}+4(q-\lambda) U_{r, x}-U_{r, x x x} .
$$

This is the standard KdV hierarchy [7]. For $r=1$, set $U_{1}(t, x, \lambda)=f_{1}(t, x) \lambda+f_{0}(t, x)$. Then

$$
\begin{aligned}
& f_{1, x}(t, x)=0, \\
& 2 q_{x}(t, x) f_{1}(t, x)-4 f_{0, x}(t, x)=0, \\
& 2 q_{t}(t, x)=2 q_{x}(t, x) f_{0}(t, x)+4 q(t, x) f_{0, x}(t, x)-f_{0, x x x}(t, x) .
\end{aligned}
$$

If $f_{1}(t, x)=c_{1}$, we obtain $c_{1} q_{x}(t, x)=2 f_{0, x}(t, x)$, which implies $f_{0}(t, x)=\frac{c_{1}}{2} q(t, x)+c_{2}$. Hence the last relation in the system above gives us

$$
q_{t}(t, x)=\frac{3}{2} c_{1} q(t, x) q_{x}(t, x)-\frac{c_{1}}{4} q_{x x x}(t, x)+c_{2} q_{x}(t, x),
$$

which is a generalized version of the classical $\mathrm{KdV}$ equation. If $c_{1}=1$ and $c_{2}=0$, we obtain the classical KdV equation, i.e.,

$$
q_{t}(t, x)=\frac{3}{2} q(t, x) q_{x}(t, x)-\frac{1}{4} q_{x x x}(t, x) .
$$

As another example, let us assume that $k=1$ and let $p(t, x)=q(t, x)=1$ be fixed. Then (17) translates to

$$
2 \lambda^{2} y_{t}(t, x)=2 \lambda y_{x}(t, x) U_{r}(t, x, \lambda)-4(1-\lambda y(t, x)) U_{r, x}(t, x, \lambda)+U_{r, x x x}(t, x, \lambda) .
$$

This is a version of the Camassa-Holm hierarchy (another one can be obtained by setting $k=r$ as in [12]). If $r=1$, a possible solution is given by

$$
\begin{aligned}
& f_{0}=c_{1}, \quad c_{1} y(t, x)+c_{2}=2 f_{1}(t, x)-\frac{1}{2} f_{1, x x}(t, x), \\
& y_{t}(t, x)=y_{x}(t, x) f_{1}(t, x)+2 y(t, x) f_{1, x}(t, x) .
\end{aligned}
$$

This system is a generalized version of the Camassa-Holm equation. The classical CamassaHolm equation is obtained by setting $c_{1}=1$ and $c_{2}=0$ (see [5]).

Again, let us set $p(t, x) \equiv \varepsilon, y(t, x) \equiv 1, k=0$ and $r=1$. Then $\tilde{U}_{1}=U_{1} / \varepsilon$, and the equation (17) translates to the system

$$
f_{1}=c_{1}, \quad c_{1} q_{x}=2 f_{0}, \quad q_{t}=\frac{3}{2} c_{1} q q_{x}-\frac{c_{1} \varepsilon}{4} q_{x x x}+c_{2} q_{x} .
$$


If $c_{1}=4$ and $c_{2}=0$, then the compatibility condition is given by

$$
q_{t}=6 q q_{x}-\varepsilon q_{x x x}
$$

which is a well-known and important generalization of the $\mathrm{KdV}$ equation, used in [28, 29, 30, $43]$ in connection with Burger's equation, which is indeed the limit as $\varepsilon \rightarrow 0$ of such a KdV generalization.

Moreover, if $p(t, x)=1, q(t, x) \equiv \varepsilon, k=1$ and $g=1$, then the compatibility condition reads (for suitably chosen constants $c_{1}$ and $c_{2}$ )

$$
4 \varepsilon u_{1, t}-u_{1, x x t}=12 \varepsilon u_{1} u_{1, x}-u_{1} u_{1, x x x}-2 u_{1, x} u_{1, x x} .
$$

This equation is a generalization of the $\mathrm{CH}$ equation. Its limit (whenever it exists) as $\varepsilon \rightarrow 0$ is the Hunter-Saxton equation

$$
u_{1, x x t}=u_{1} u_{1, x x x}+2 u_{1, x} u_{1, x x} .
$$

Note that the constants in all the above constructions can be chosen at will.

Before proceeding with the discussion, we wish to make another observation: the fact that both the KdV and the Camassa-Holm hierarchies are included in our hierarchy is not surprising at all. Indeed, they are strictly related as one can use a Liouville transform to move from one hierarchy to the other $[21,38]$.

The answer to Question 2 is affirmative. In more detail, at first we choose (at will!!) a family $(p(t, x), \mathcal{M}(t, x)) \in \mathcal{E}_{2}$. We then construct a polynomial $U_{r}$ in the following way: the coefficients of $U_{r}$ are determined recursively via the relation

$$
\tilde{U}_{r, x}(\lambda)=\frac{\lambda^{k}}{p}\left[\frac{\mathcal{M}_{t}}{\mathcal{M}}+p\left(\frac{1}{p}\right)_{t}\right]-\frac{\mathcal{M}_{x}}{\mathcal{M}} \tilde{U}_{r}(\lambda)+\sum_{i=0}^{n}\left[\frac{\lambda^{k}}{P_{i}^{k}} \tilde{U}_{r}\left(P_{i}\right)-\tilde{U}_{r}(\lambda)\right] \frac{\lambda P_{i, x}}{P_{i}\left(\lambda-P_{i}\right)},
$$

where we omitted to write down explicitly the dependence of the functions with respect to $t$ and $x$. Note that $p$ and $\mathcal{M}$ are known functions of $(t, x)$, while the functions (poles) $P_{i}=P_{i}(t, x)$ remain to be determined.

Once this is done, we determine the poles $P_{1}(t, x), \ldots, P_{g}(t, x)$ by solving the system

$$
\begin{aligned}
& P_{i, x}(t, x)=\frac{-\mathcal{M}(t, x) k_{g}\left(P_{i}(t, x)\right) \prod_{i=1}^{g} P_{i}(t, x)}{p(t, x) k_{g}\left(0^{+}\right) \prod_{j \neq i}\left(P_{j}(t, x)-P_{i}(t, x)\right)} \quad(\text { as in }(6)), \\
& P_{i, t}(t, x)=\frac{U_{r}\left(t, x, P_{i}(t, x)\right)}{P_{i}^{k}(t, x)} P_{i, x}(t, x)
\end{aligned}
$$

together with the initial condition $P_{1}(0,0) \in\left[\lambda_{1}, \lambda_{2}\right], \ldots, P_{g}(0,0) \in\left[\lambda_{2 g-1}, \lambda_{2 g}\right]$. Then it turns out that the system (20) is consistent, and that the polynomial $U_{r}(t, x, \lambda)$ gives rise, via the corresponding matrix $B_{r}$, to a solution of (16).

Moreover, one can write down the trace formulas (analogous to those in (7) and (8)):

$$
\begin{aligned}
y(t, x) & =\frac{\mathcal{M}^{2}(t, x) \prod_{i=1}^{g} P_{i}^{2}(t, x)}{4 p(t, x) k^{2}\left(0^{+}\right)} \\
q(t, x) & =y(t, x)\left(\lambda_{0}+\sum_{i=1}^{g} \lambda_{2 i-1}+\lambda_{2 i}-2 P_{i}(t, x)\right)+\tilde{q}(t, x),
\end{aligned}
$$


where

$$
\tilde{q}(t, x)=-\left(\frac{(p(t, x) y(t, x))_{x}}{4 y(t, x)}\right)_{x}+\left(\frac{(p(t, x) y(t, x))_{x}}{4 y(t, x)}\right)^{2} .
$$

The functions $q(t, x)$ and $y(t, x)$ are the solutions of the evolution equations (18), hence we have solved the Sturm-Liouville evolution equation of order $r$. Notice that, since the maps $x \mapsto P_{i}(t, x)$ satisfy the first equation in the system $(20)$, the triple $a(t, \cdot)=(p(t, \cdot), q(t, \cdot), y(t, \cdot))$ lies in the isospectral class of the algebro-geometric potential $a(0, \cdot)$ for every $t \in \mathbb{R}$, hence the map $t \mapsto a(t, x)$ is a curve in the isospectral class of $a(0, x)$ starting from $a(0, x)$ !

Let us further repeat that these developments can be carried out both in the case when $U_{g}$ contains the factors $E_{i}$ and $\frac{1}{\lambda_{*}-\lambda}$ and in the case when these factors are not present.

\section{Some solutions of the Sturm-Liouville hierarchy}

All the machinery we have discussed in the previous section works well when we take as initial conditions potentials of algebro-geometric type. What happens if we change the initial condition? Clearly, we cannot choose an initial condition at will, because the structure of the hierarchy has to remain consistent. In particular, the structure of the function $U_{g}$ must be preserved in some sense. An idea is that of considering as initial data some reflectionless Sturm-Liouville potentials whose spectra consist of infinitely many intervals clustering at $\infty$. This has been done in [24]. In this case $U_{g}$ translates to an entire function $U(t, x)$ with the infinitely many zeros $P_{1}(t, x), \ldots, P_{g}(t, x), \ldots$ It is important, however, that instead $U_{r}$ remain a polynomial of degree $r$. The reader can be addressed to [23, 24] for a detailed discussion of these topics.

The purpose of this section is that of enlarging the class of initial conditions for which the Sturm-Liouville hierarchy can be solved, by including other reflectionless potentials whose spectra can cluster at a finite real point $\lambda_{*}$. The discussion of these new potentials will require the introduction of the factors $E_{i}$ and $\frac{1}{\lambda_{*}-\lambda}$ seen in the definition of $U_{g}$ and $\tilde{k}_{g}$ given in (9) and (10) respectively.

Before introducing a suitable hierarchy of evolution equations, or rather a zero-curvature relation which determines such a hierarchy, we should explain how to construct reflectionless potentials with some prescribed properties of the spectrum of the associated operator. We will use a procedure which we call of algebro-geometric approximation. The construction we are going to illustrate is described in detail in [24] in the case when $\lambda_{*}=\infty$. Let us fix a sequence of positive real numbers

$$
\tilde{\Lambda}=\left\{\lambda_{0}<\lambda_{1}<\lambda_{2}<\cdots<\lambda_{2 g}<\cdots\right\} .
$$

Set $I_{k}=\left[\lambda_{2 k-1}, \lambda_{2 k}\right], h_{i j}=\operatorname{dist}\left(I_{i}, I_{j}\right), d_{j}=\lambda_{2 j}-\lambda_{2 j-1}$ and $h_{0 k}=\lambda_{2 k-1}-\lambda_{0}$. We assume that the sequence $\tilde{\Lambda}$ satisfies the following assumptions:

$$
\begin{aligned}
& \text { (H1) } \lim _{i \rightarrow \infty} \lambda_{i}=\lambda_{*}, \\
& \text { (H2) } \sum_{j=1}^{\infty} d_{j}<\infty, \\
& \text { (H3) } \sup _{j \in \mathbb{N}} \sum_{k \neq j} \frac{\sqrt{d_{k}}}{h_{j k}}<\infty .
\end{aligned}
$$

We will construct a Sturm-Liouville potential $a(x)=(p(x), q(x), y(x)) \in \mathcal{E}_{3}$ which is reflectionless and such that the spectrum of the associated operator $L_{a}$ is given by

$$
\Sigma=\left[\lambda_{0}, \lambda_{1}\right] \cup\left[\lambda_{2}, \lambda_{3}\right] \cup \cdots \cup\left[\lambda_{2 g}, \lambda_{2 g+1}\right] \cup \cdots \cup\left[\lambda_{*}, \infty\right) .
$$


Actually, the method we will describe below can be applied to prove the existence of a reflectionless Sturm-Liouville potential such that the spectrum of the associated Sturm-Liouville operator is given by

$$
\Sigma=\bigcap_{g \in \mathbb{N}} \Sigma_{g}
$$

where

$$
\Sigma_{g}=\left[\lambda_{0}, \lambda_{1}\right] \cup \cdots \cup\left[\lambda_{2 g}, \lambda_{*}\right] \cup[\bar{\lambda}, \infty),
$$

and $\bar{\lambda}$ is any real number strictly greater than $\lambda_{*}$. Also, this method can be applied when there is more than one cluster point in the sequence $\left\{\lambda_{i}\right\}$, and in fact when there is an arbitrary finite number of cluster points $\left\{\lambda_{*}^{(1)}, \ldots, \lambda_{*}^{(k)}\right\}$.

However, to keep the discussion clearer, we will only deal with the case when

$$
\Sigma=\left[\lambda_{0}, \lambda_{1}\right] \cup\left[\lambda_{2}, \lambda_{3}\right] \cup \cdots \cup\left[\lambda_{2 g}, \lambda_{2 g+1}\right] \cup \cdots \cup\left[\lambda_{*}, \infty\right) .
$$

The procedure is inspired by the following important proposition [17] (see also [20, 23, 24]).

Proposition 1. Let $\left\{a_{n}\right\}=\left\{\left(p_{n}, q_{n}, y_{n}\right)\right\} \subset \mathcal{E}_{3}$ be a sequence of potentials such that $a_{n} \rightarrow$ $a=(p, q, y) \in \mathcal{E}_{3}$ uniformly on compact subsets of $\mathbb{R}$. Assume that $a_{n}$ is reflectionless and that $\Sigma_{a_{n+1}} \subset \Sigma_{a_{n}}$ for every $n \in \mathbb{N}$. Assume further that the set $\Sigma=\bigcap_{n \in \mathbb{N}} \Sigma_{a_{n}}$ has locally positive Lebesgue measure. Then a is reflectionless and the spectrum $\Sigma_{a}$ of the operator $L_{a}$ equals the set $\Sigma$.

We will not prove this proposition. It uses the Weyl decreasing disc construction and some additional reasoning concerning the spectral measures and the spectra of the operators $L_{a_{n}}$.

Inspired by the above proposition, we fix the finite set $\tilde{\Lambda}_{g} \subset \tilde{\Lambda}$ given by

$$
\tilde{\Lambda}_{g}=\left\{\lambda_{0}, \lambda_{1}, \ldots, \lambda_{2 g}\right\} \text {, }
$$

then choose points $P_{j}(0) \in\left[\lambda_{2 j-1}, \lambda_{2 j}\right], j=1, \ldots, g$. Moreover, let us fix a pair $(p(x), \mathcal{M}(x)) \in \mathcal{E}_{2}$. In correspondence with these choices, one can construct an algebro-geometric potential $a_{g}=$ $\left(p(x), q_{g}(x), y_{g}(x)\right) \in \mathcal{E}_{3}$ such that the spectrum of the operator $L_{a_{g}}$ is given by

$$
\Sigma_{g}=\left[\lambda_{0}, \lambda_{1}\right] \cup \cdots \cup\left[\lambda_{2 g}, \infty\right),
$$

and such that the trace formulas (7) and (8) hold, together with the system (6). Now we let $g$ vary over $\mathbb{N}$. We obtain sequences $\left\{a_{g}\right\}=\left\{p, q_{g}, y_{g}\right\} \in \mathcal{E}_{3}$ of algebro-geometric potentials and corresponding poles $\left\{P_{j}^{(g)}(x)\right\}$. Next, we let $g \rightarrow \infty$. It can be shown that the sequences $\left\{P_{j}^{(g)}(x)\right\} \rightarrow\left\{P_{j}(x)\right\}$ for every $x \in \mathbb{R}$, and that $a_{g} \rightarrow a=(p, q, y) \in \mathcal{E}_{3}$ uniformly on compact subsets of $\mathbb{R}$ (this convergence, however is not uniform on $\mathbb{R}[20,24]$ ). One can show that the poles $P_{j}(x)$ satisfy the following system of infinitely many ODE's $(j \in \mathbb{N})$

$$
\begin{aligned}
P_{j, x}(x)= & \pm \frac{\mathcal{M}(x)}{\sqrt{\lambda_{0}} p(x)}\left(\prod_{k \in \mathbb{N}} \frac{P_{k}(x)}{\sqrt{\lambda_{2 k-1} \lambda_{2 k}}}\right) \sqrt{\left(\lambda_{2 j}-P_{j}(x)\right)\left(P_{j}(x)-\lambda_{2 j-1}\right)} \\
& \times\left(\prod_{k \neq j} \frac{\sqrt{\left(\lambda_{2 k-1}-P_{j}(x)\right)\left(\lambda_{2 k}-P_{j}(x)\right)}}{P_{k}(x)-P_{j}(x)}\right) \sqrt{P_{j}(x)-\lambda_{0}} .
\end{aligned}
$$


The sign \pm in the equations (21) comes from the necessity to choose a sign of the square root $\sqrt{\left(\lambda_{2 k-1}-P_{j}(x)\right)\left(\lambda_{2 k}-P_{j}(x)\right)}$. This ambiguity, however, can be avoided by passing to suitable angular coordinates $\theta_{1}(x), \ldots, \theta_{n}(x), \ldots$ But this is not the place in which to discuss this matter.

Once we have determined the pole motion, we can write the trace formulas

$$
\begin{aligned}
& y(x)=\frac{\mathcal{M}^{2}(x)}{4 p(x) \lambda_{0}} \prod_{k \in \mathbb{N}} \frac{P_{k}^{2}(x)}{\lambda_{2 k-1} \lambda_{2 k}}, \\
& q(x)=y(x)\left(\lambda_{0}+\sum_{k \in \mathbb{N}} \lambda_{2 k-1}+\lambda_{2 k}-2 P_{k}(x)\right)+\tilde{q}(x),
\end{aligned}
$$

where

$$
\tilde{q}(x)=-\left(\frac{(p(x) y(x))_{x}}{4 y(x)}\right)_{x}+\left(\frac{(p(x) y(x))_{x}}{4 y(x)}\right)^{2} .
$$

The assumptions (H1)-(H3) are used to show that the appropriate quantities are well defined and converge properly. See [20, 24] for the above developments.

Now we move to the main question of interest in this paper, namely the solution of the Sturm-Liouville hierarchy for certain non algebro-geometric reflectionless initial data.

First, we introduce a zero-curvature relation which takes into account the structure of the potential $a$ obtained above. To do this, let the family $\{(p(t, x), \mathcal{M}(t, x))\} \in \mathcal{E}_{2}$ (indexed by $t \in \mathbb{R}$ ) be fixed. Choose the set $\tilde{\Lambda}$ as above and initial data $P_{1}(t, 0) \in\left[\lambda_{1}, \lambda_{2}\right], \ldots, P_{g}(t, 0) \in\left[\lambda_{2 g-1}, \lambda_{2 g}\right], \ldots$ in such a way that they vary smoothly with respect to $t \in \mathbb{R}$. Let $P_{1}^{(g)}(t, x), \ldots, P_{g}^{(g)}(t, x)$ be the solution of the system

$$
P_{i, x}^{(g)}(t, x)=\frac{-\mathcal{M}(t, x) k_{g}\left(P_{i}^{(g)}(t, x)\right) \prod_{i=1}^{g} P_{i}^{(g)}(t, x)}{p(t, x) k_{g}\left(0^{+}\right) \prod_{j \neq i}\left(P_{j}^{g)}(t, x)-P_{i}^{(g)}(t, x)\right)} .
$$

For every fixed $t \in \mathbb{R}$, let us construct the sequence $\left\{a_{g}(t, x)=\left(p(t, x), q_{g}(t, x), y_{g}(t, x)\right)\right\} \subset \mathcal{E}_{3}$ as above, and let $a(t, x)=(p(t, x), q(t, x), y(t, x))$ be its limit in $\mathcal{E}_{3}$ (we emphasize that the variable here is $x$, while $t$ is considered as a parameter). Let

$$
U_{g}(t, x, \lambda)=\frac{-2 p(t, x) k_{g}\left(0^{+}\right)}{\mathcal{M}(t, x) \prod_{i=1}^{g} P_{i}^{(g)}(t, x)} \prod_{i=1}^{g} \frac{\lambda-P_{i}^{(g)}(t, x)}{\lambda_{*}-\lambda} E_{i}\left(\frac{\lambda_{*}-\lambda_{2 i}}{\lambda_{*}-\lambda}\right),
$$

where

$$
E_{n}(\lambda)=\exp \left(\lambda+\frac{\lambda^{2}}{2}+\cdots+\frac{\lambda^{n}}{n}\right) .
$$

The function $\lambda \mapsto U_{g}(t, x, \lambda)$ is defined in the region $G=\mathbb{C} \backslash\left\{\lambda_{*}\right\}$.

We prove the following

Theorem 3. As $g \rightarrow \infty$ the functions $U_{g}(t, x, \lambda)$ converge to a holomorphic function $U(t, x, \lambda)$, uniformly on compact subsets of $G$. This convergence is uniform also with respect to $(t, x) \in \mathbb{R}^{2}$.

Proof. For every fixed $t \in \mathbb{R}$, the poles $P_{j}^{(g)}(t, x)$ converge pointwise to poles $P_{j}(t, x)$ as $g \rightarrow \infty$, where $P_{j}(t, x)$ satisfy the relation $(21)(j \in \mathbb{N})$. Each $P_{j}(t, x)$ lies in the corresponding interval $I_{j}=\left[\lambda_{2 j-1}, \lambda_{2 j}\right]$ for every $(t, x) \in \mathbb{R}^{2}$. 
Now, if $g \rightarrow \infty$, the pointwise limit of $U_{g}(t, x, \lambda)$ is given by the function

$$
U(t, x, \lambda)=-\frac{2 \sqrt{\lambda_{0}} p(t, x)}{\mathcal{M}(t, x)} \prod_{k=1}^{\infty} \frac{\sqrt{\lambda_{2 k-1} \lambda_{2 k}}}{P_{k}(t, x)} \prod_{i=1}^{\infty} \frac{\lambda-P_{i}(t, x)}{\lambda_{*}-\lambda} E_{i}\left(\frac{\lambda_{*}-\lambda_{2 i}}{\lambda_{*}-\lambda}\right) .
$$

However, the expression (22) has only informal significance at the moment, because we do not know if it exists (the infinite products must converge properly!).

The infinite product

$$
\prod_{k=1}^{\infty} \frac{\sqrt{\lambda_{2 k-1} \lambda_{2 k}}}{P_{k}(t, x)}
$$

is well defined, because

$$
\prod_{k=1}^{\infty} \frac{\sqrt{\lambda_{2 k-1} \lambda_{2 k}}}{P_{k}(t, x)} \leq \prod_{k=1}^{\infty} \frac{\lambda_{2 k}}{P_{k}(t, x)}
$$

and the series

$$
\sum_{k=1}^{\infty}\left|1-\frac{\lambda_{2 k}}{P_{k}(t, x)}\right| \leq \sum_{k=1}^{\infty}\left|\frac{d_{k}}{h_{0 k}}\right|
$$

converges, using the assumption (H2), uniformly with respect to $(t, x) \in \mathbb{R}^{2}$. Hence, the main problem lies in proving that the infinite product

$$
\prod_{i=1}^{\infty} \frac{\lambda-P_{i}(t, x)}{\lambda_{*}-\lambda} E_{i}\left(\frac{\lambda_{*}-\lambda_{2 i}}{\lambda_{*}-\lambda}\right)
$$

exists. Observe that, if the factors $E_{i}$ and $\frac{1}{\lambda_{*}-\lambda}$ are absent, the convergence does not hold. Our use of these factors is motivated by the classical theory of Weierstrass and Runge [26].

To prove this, let $K \subset G$ be a compact subset. We claim that the series

$$
\sum_{i=1}^{\infty}\left|1-\frac{\lambda-P_{i}(t, x)}{\lambda_{*}-\lambda} E_{i}\left(\frac{\lambda_{*}-\lambda_{2 i}}{\lambda_{*}-\lambda}\right)\right|
$$

converges uniformly with respect to $\lambda \in K$ and $(t, x) \in \mathbb{R}^{2}$. By a well-known result on infinite products, this will imply that the infinite product under consideration is well defined. Let us rewrite

$$
\begin{aligned}
\sum_{i=1}^{\infty} \mid & 1-\frac{\lambda-P_{i}(t, x)}{\lambda_{*}-\lambda} E_{i}\left(\frac{\lambda_{*}-\lambda_{2 i}}{\lambda_{*}-\lambda}\right)\left|\leq \sum_{i=1}^{\infty}\right| 1-\frac{\lambda-\lambda_{2 i}}{\lambda_{*}-\lambda} E_{i}\left(\frac{\lambda_{*}-\lambda_{2 i}}{\lambda_{*}-\lambda}\right) \mid \\
& +\sum_{i=1}^{\infty}\left|\frac{\lambda_{2 i}-P_{i}(t, x)}{\lambda_{*}-\lambda} E_{i}\left(\frac{\lambda_{*}-\lambda_{2 i}}{\lambda_{*}-\lambda}\right)\right|
\end{aligned}
$$

Let $D=\operatorname{dist}(\mathbb{C} \backslash G, K)$. If $\lambda \in K$, then

$$
\left|\frac{\lambda_{*}-\lambda_{2 i}}{\lambda_{*}-\lambda}\right| \leq \frac{\left|\lambda_{*}-\lambda_{2 i}\right|}{D} .
$$

Since $\lambda_{2 i} \rightarrow \lambda_{*}$, for every $0<\varepsilon<1$, there holds

$$
\left|\frac{\lambda_{*}-\lambda_{2 i}}{\lambda_{*}-\lambda}\right| \leq \varepsilon
$$

for sufficiently large $i \in \mathbb{N}$. 
Now, the factors of the second summand in the r.h.s. of (23) can be estimated as follows:

$$
\left|\frac{\lambda_{2 i}-P_{i}(t, x)}{\lambda_{*}-\lambda} E_{i}\left(\frac{\lambda_{*}-\lambda_{2 i}}{\lambda_{*}-\lambda}\right)\right| \leq \frac{d_{i}}{D} \exp \left(\varepsilon+\frac{\varepsilon}{2}+\cdots+\frac{\varepsilon^{i}}{i}\right)
$$

for sufficiently large $i \in \mathbb{N}$. Since the series $\sum_{i=1}^{\infty} \frac{\varepsilon^{i}}{i}=D_{1}<\infty$, the second summand in the r.h.s. of (23) is dominated by the uniformly convergent series

$$
\frac{e^{D_{1}}}{D} \sum_{i=1}^{\infty} d_{i}<\infty
$$

We now move our attention to the first summand in the r.h.s. of (23). To prove that it converges, we can argue as follows. Let us set $z=\frac{\lambda_{*}-\lambda_{2 i}}{\lambda_{*}-\lambda}$. Then

$$
\frac{\lambda-\lambda_{2 i}}{\lambda_{*}-\lambda} E_{i}\left(\frac{\lambda_{*}-\lambda_{2 i}}{\lambda_{*}-\lambda}\right)=(z-1) E_{i}(z)=F_{i}(z) .
$$

We expand $F_{i}(z)$ at $z=0$, so that

$$
F_{i}(z)=1+\sum_{k=1}^{\infty} a_{k} z^{k}
$$

Differentiating $F_{i}(z)$ with respect to $z$, we obtain

$$
\sum_{k=1}^{\infty} k a_{k} z^{k-1}=-z^{i} E_{i}(z) .
$$

Also $E_{i}(z)$ can be expanded at $z=0$, being an exponential function. Doing so, one observes that $a_{1}, \ldots, a_{i}=0$. Moreover, since the coefficients of the expansion of $E_{i}(z)$ are all positive, we must have

$$
\left|a_{k}\right|=-a_{k}
$$

for every $k>i$. This implies that

$$
0=F_{i}(1)=1+\sum_{k=i+1}^{\infty} a_{k}
$$

hence

$$
\sum_{k=i+1}^{\infty}\left|a_{k}\right|=1
$$

But now we have

$$
\left|1-F_{i}(z)\right| \leq|z|^{i+1}\left(\sum_{k=i+1}^{\infty}\left|a_{k}\right|\right)=|z|^{i+1},
$$

whenever $|z| \leq 1$. Since $|z|=\left|\frac{\lambda_{*}-\lambda_{2 i}}{\lambda_{*}-\lambda}\right| \leq \frac{\varepsilon}{D}<1$ for sufficiently large $i \in \mathbb{N}$, we have

$$
\sum_{i=1}^{\infty}\left|1-\frac{\lambda-\lambda_{2 i}}{\lambda_{*}-\lambda} E_{i}\left(\frac{\lambda_{*}-\lambda_{2 i}}{\lambda_{*}-\lambda}\right)\right| \leq \sum_{i=1}^{\infty}\left(\frac{\varepsilon}{D}\right)^{i+1}<\infty .
$$

We proved that the whole series of the l.h.s. of (23) is dominated by a uniformly convergent series, whenever $\lambda \in K$ and $(t, x) \in \mathbb{R}^{2}$. By standard facts concerning infinite products, the theorem is proved. 
Now, we prove the following

Lemma 1. Let $K \subset G$ be a compact subset, say $K=\{\lambda \in G|| \lambda \mid<l\}$. Then the functions $x \mapsto U_{g, x}(t, x, \lambda)$ are uniformly bounded in $\mathbb{R}$, for $\lambda \in K$ and uniformly in $t \in \mathbb{R}$. Hence the maps $x \mapsto U_{g}(t, x, \lambda)$ converge uniformly on compact subsets of $\mathbb{R}$ to $U(t, x, \lambda)$ for $\lambda \in K$ and $t \in \mathbb{R}$.

Proof. The derivative $U_{g, x}(t, x, \lambda)$ can be estimated as follows:

$$
\left|U_{g, x}\right| \leq \alpha\left|U_{g}\right|+\frac{2 p}{\mathcal{M}} \frac{k_{g}\left(0^{+}\right)}{\prod_{j=1}^{g} P_{j}^{(g)}}\left(\sum_{j=1}^{g} \frac{\left|l P_{j, x}^{(g)}\right|}{\left(P_{j}^{(g)}\right)^{2}} \prod_{k \neq j}\left(\frac{\lambda-P_{k}^{(g)}}{\lambda-\lambda_{*}}\right) E_{k}\left(\frac{\lambda_{*}-\lambda_{2 k}}{\lambda_{*}-\lambda}\right)\right),
$$

where $\alpha=\sup _{x \in \mathbb{R}}\left|\frac{p_{x}}{p}-\frac{\mathcal{M}_{x}}{\mathcal{M}}\right|$. Since $U_{g}$ is uniformly bounded, the only thing to check is that

$$
\sum_{j=1}^{\infty} \frac{\left|P_{j, x}^{(g)}\right|}{\left(P_{j}^{(g)}\right)^{2}}
$$

converges, because

$$
\left|\prod_{k \neq j}\left(\frac{\lambda-P_{k}^{(g)}}{\lambda-\lambda_{*}}\right) E_{k}\left(\frac{\lambda_{*}-\lambda_{2 k}}{\lambda_{*}-\lambda}\right)\right|
$$

is bounded uniformly with respect to $g \in \mathbb{N}$ and $(t, x) \in \mathbb{R}^{2}$, whenever $\lambda \in K$.

It can be shown (see $[20,23,24])$ that

$$
\left|P_{j, x}^{(g)}(t, x)\right| \leq C \sqrt{d_{j}}
$$

where $C$ does not depend on $g \in \mathbb{R}$ and $(t, x) \in \mathbb{R}^{2}$, hence the series

$$
\sum_{j=1}^{\infty} \frac{\left|P_{j, x}^{(g)}\right|}{\left(P_{j}^{(g)}\right)^{2}} \leq C \sum_{j=1}^{\infty} \frac{\sqrt{d_{j}}}{h_{0 j}^{2}}<\infty
$$

The lemma is proved.

Define the matrix $B_{g}(t, x, \lambda)$ as in Section 3. This matrix has entries depending on $U_{g}$ and two more functions $T_{g}$ and $V_{g}$ defined so as to satisfy (11) and (12) respectively. Moreover the equation (13) and the stationary zero-curvature relation $-B_{g, x}+\left[A, B_{g}\right]=0$ hold.

Since $\left\{U_{g}\right\}$ and $\left\{U_{g, x}\right\}$ are uniformly bounded on every compact subset of $\mathbb{R}$, it follows that $\left\{T_{g}\right\}$ is uniformly bounded on every compact subset of $\mathbb{R}$. Using (12) and (13), one easily shows that $\left\{V_{g}\right\}$ is uniformly bounded on each compact subset of $\mathbb{R}$ as well. This tells us that: (1) the matrices $B_{g}$ converge to a matrix $B$ in every compact subset $K \subset G$ and uniformly on compact subsets of $\mathbb{R}^{2} ;(2)$ writing the stationary zero-curvature relation as

$$
B_{g}(t, x, \lambda)-B_{g}(t, 0, \lambda)=\int_{0}^{x}\left[A_{g}(t, s, \lambda), B_{g}(t, s, \lambda)\right] d s,
$$

we can use the bounded convergence theorem to conclude that also

$$
B(t, x, \lambda)-B(t, 0, \lambda)=\int_{0}^{x}[A(t, s, \lambda), B(t, s, \lambda)] d s
$$


i.e.,

$$
-B_{x}(t, x, \lambda)+[A(t, x, \lambda), B(t, x, \lambda)]=0 .
$$

Now we specify the $t$-dependence of $a(t, x)=(p(t, x), q(t, x), y(t, x))$, as follows. Fix a number $r \in \mathbb{N}$, and introduce a matrix $B_{r}$ as in Section 3. Again, we introduce the polynomial

$$
U_{r}(t, x, \lambda)=\sum_{j=0}^{r} p(t, x) f_{j}(t, x) \lambda^{j} .
$$

In correspondence with every $0 \leq r<g$, there exists a matrix $B_{r}^{(g)}(t, x, \lambda)$, together with a polynomial $U_{r}^{(g)}$ and functions $T_{r}^{(g)}$ and $V_{r}^{(g)}$ satisfying the system (16). The polynomial $U_{r}^{(g)}$ can be found recursively using the relation (19). The corresponding poles $P_{1}^{(g)}(t, x), \ldots, P_{g}^{(g)}(t, x)$ solve the system

$$
\begin{aligned}
P_{i, x}^{(g)}(t, x) & =\frac{-\mathcal{M}(t, x) k_{g}\left(P_{i}^{(g)}(t, x)\right) \prod_{i=1}^{g} P_{i}^{(g)}(t, x)}{p(t, x) k_{g}\left(0^{+}\right) \prod_{j \neq i}\left(P_{j}^{(g)}(t, x)-P_{i}^{(g)}(t, x)\right)} \quad(\text { as in }(6)), \\
P_{i, t}^{(g)}(t, x) & =\frac{U_{r}^{(g)}\left(t, x, P_{i}^{(g)}(t, x)\right)}{\left(P_{i}^{(g)}(t, x)\right)^{k}} P_{i, x}^{(g)}(t, x) .
\end{aligned}
$$

A direct analysis shows that each coefficient of the polynomial $U_{r}^{(g)}$ is well defined, since it is a linear combination of at most $r$ symmetric functions of the poles $P_{1}^{(g)}(t, x), \ldots, P_{g}^{(g)}(t, x)$, plus possibly a uniformly bounded function. To make this clearer, we write down the form of the first of these coefficients when $k=0$ (the case when $k \neq 0$ is similar). For fixed $t \in \mathbb{R}$, set

$$
H_{g}(t, x)=\frac{\mathcal{M}(t, 0)}{\mathcal{M}(t, x)} \prod_{i=1}^{g} \frac{P_{i}^{(g)}(t, 0)}{P_{i}^{(g)}(t, x)} .
$$

We have

$$
\begin{aligned}
f_{r}^{(g)}= & c_{r}^{(g)} H_{g}(t, x), \\
f_{r-1}^{(g)}= & H_{g}(t, x)\left[c_{r-1}^{(g)}+c_{r}^{(g)}\left(\sum_{i=1}^{g} P_{i}^{(g)}(t, x)-P_{i}^{(g)}(t, 0)\right)\right], \\
f_{r-2}^{(g)}= & H_{g}(t, x)\left[c_{r-2}^{(g)}-c_{r-1}^{(g)}\left(\sum_{i=1}^{g}\left(P_{i}^{(g)}(t, x)-P_{i}^{(g)}(t, 0)\right)\right)\right. \\
& \left.+c_{r}^{(g)}\left(\sum_{i<j}^{g}\left(P_{i}^{(g)}(t, x)-P_{i}^{(g)}(t, 0)\right)\left(P_{j}^{(g)}(t, x)-P_{j}^{(g)}(t, 0)\right)\right)\right],
\end{aligned}
$$

These formulas imply that the coefficients $f_{j}^{(g)}(t, x)$ converge, as $g \rightarrow \infty$, to coefficients $f_{j}(t, x)$ uniformly on compact subsets of $\mathbb{R}^{2}(1 \leq j \leq r)$, hence a polynomial $U_{r}(t, x, \lambda)$ is well defined as the limit, as $g \rightarrow \infty$, of the polynomials $U_{r}^{(g)}(t, x, \lambda)$. Using the same arguments as those applied in the discussion of $B(t, x, \lambda)$, there is a well defined matrix $B_{r}(t, x, \lambda)$ which can be obtained as the limit, as $g \rightarrow \infty$, of the matrices $B_{r}^{(g)}(t, x, \lambda)$. Using the bounded convergence theorem again, we conclude that the zero-curvature relation

$$
A_{t}(t, x, \lambda)-B_{r, x}(t, x, \lambda)+\left[A(t, x, \lambda), B_{r}(t, x, \lambda)\right]=0
$$


holds, i.e., the Sturm-Liouville hierarchy is solved, and has solutions $q(t, x)$ and $y(t, x)$ such that

$$
\begin{aligned}
& y(t, x)=\frac{\mathcal{M}^{2}(t, x)}{4 p(t, x) \lambda_{0}} \prod_{k \in \mathbb{N}} \frac{P_{k}^{2}(t, x)}{\lambda_{2 k-1} \lambda_{2 k}} \\
& q(t, x)=y(t, x)\left(\lambda_{0}+\sum_{k \in \mathbb{N}} \lambda_{2 k-1}+\lambda_{2 k}-2 P_{k}(t, x)\right)+\tilde{q}(t, x),
\end{aligned}
$$

where

$$
\tilde{q}(t, x)=-\left(\frac{(p(t, x) y(t, x))_{x}}{4 y(t, x)}\right)_{x}+\left(\frac{(p(t, x) y(t, x))_{x}}{4 y(t, x)}\right)^{2} .
$$

The poles $P_{1}(t, x), \ldots, P_{g}(t, x), \ldots$ move according to

$$
\begin{aligned}
P_{j, x}(t, x)= & \pm \frac{\mathcal{M}(t, x)}{\sqrt{\lambda_{0}} p(t, x)}\left(\prod_{k \in \mathbb{N}} \frac{P_{k}(t, x)}{\sqrt{\lambda_{2 k-1} \lambda_{2 k}}}\right) \sqrt{\left(\lambda_{2 j}-P_{j}(t, x)\right)\left(P_{j}(t, x)-\lambda_{2 j-1}\right)} \times \\
& \times\left(\prod_{k \neq j} \frac{\sqrt{\left(\lambda_{2 k-1}-P_{j}(t, x)\right)\left(\lambda_{2 k}-P_{j}(t, x)\right)}}{P_{k}(t, x)-P_{j}(t, x)}\right) \sqrt{P_{j}(x)-\lambda_{0}}
\end{aligned}
$$

and

$$
P_{j, t}(t, x)=\frac{U_{r}\left(t, x, P_{j}(t, x)\right)}{P_{j}^{k}(t, x)} P_{j, x}(t, x),
$$

where $U_{r}(t, x, \lambda)$ is defined as the (pointwise) limit as $g \rightarrow \infty$ of the polynomial $U_{r}^{(g)}(t, x, \lambda)$.

\section{References}

[1] Alber M.S., Fedorov Y.N., Algebraic geometrical solutions for certain evolution equations and Hamiltonian flows on nonlinear subvarieties of generalized Jacobians, Inverse Problems 17 (2001), 1017-1042.

[2] Beals R., Sattinger D.H., Szmigielski J., Multipeakons and the classical moment problem, Adv. Math. 154 (2000), 229-257, solv-int/9906001.

[3] Belokolos E.D., Bobenko A.I., Enol'skii V.Z., Its A.R., Matveev V.B., Algebro-geometric approach to nonlinear integrable equations, Springer Series in Nonlinear Dynamics, Springer-Verlag, Berlin, 1994.

[4] Boutet de Monvel A., Egorova I., On solutions of nonlinear Schrödinger equations with Cantor-type spectrum, J. Anal. Math. 72 (1997), 1-20.

[5] Camassa R., Holm D.D., An integrable shallow water equation with peaked solitons, Phys. Rev. Lett. 71 (1993), 1661-1664, patt-sol/9305002.

[6] Craig W., The trace formula for Schrödinger operators on the line, Comm. Math. Phys. 126 (1989), 379-407.

[7] Dubrovin B.A., Matveev V.B., Novikov S.P., Nonlinear equations of Korteweg-de Vries type, finite-band linear operators and Abelian varieties, Russ. Math. Surv. 31 (1976), 59-146.

[8] Egorova I.E., On a class of almost periodic solutions of the KdV equation with a nowhere dense spectrum, Russian Acad. Sci. Dokl. Math. 45 (1993), 290-293.

[9] Egorova I.E., The Cauchy problem for the KdV equation with almost periodic initial data whose spectrum is nowhere dense, in Spectral operator theory and related topics, Adv. Soviet Math., Vol. 19, Amer. Math. Soc., Providence, RI, 1994, 181-208.

[10] Fabbri R., Johnson R., Zampogni L., Nonautonomous differential systems in two dimensions, in Handbook of Differential Equations: Ordinary Differential Equations, Vol. IV, Handb. Differ. Equ., Elsevier/NorthHolland, Amsterdam, 2008, 133-268.

[11] Gardner C.S., Greene J.M., Kruskal M.D., Miura R.M., Method for solving the Korteweg-de Vries equation, Phys. Rev. Lett. 19 (1967), 1095-1097. 
[12] Gesztesy F., Holden H., Algebro-geometric solutions of the Camassa-Holm hierarchy, Rev. Mat. Iberoam. 19 (2003), 73-142, nlin.SI/0105021.

[13] Gesztesy F., Holden H., Soliton equations and their algebro-geometric solutions. Vol. I. (1+1)-dimensional continuous models, Cambridge Studies in Advanced Mathematics, Vol. 79, Cambridge University Press, Cambridge, 2003.

[14] Gesztesy F., Karwowski W., Zhao Z., Limits of soliton solutions, Duke Math. J. 68 (1992), 101-150.

[15] Johnson R., Exponential dichotomy, rotation number, and linear differential operators with bounded coefficients, J. Differential Equations 61 (1986), 54-78.

[16] Johnson R., On the Sato-Segal-Wilson solutions of the K-dV equation, Pacific J. Math. 132 (1988), 343355.

[17] Johnson R., Moser J., The rotation number for almost periodic potentials, Comm. Math. Phys. 84 (1982), 403-438.

[18] Johnson R., Zampogni L., On the inverse Sturm-Liouville problem, Discrete Contin. Dyn. Syst. 18 (2007), 405-428.

[19] Johnson R., Zampogni L., Description of the algebro-geometric Sturm-Liouville coefficients, J. Differential Equations 244 (2008), 716-740.

[20] Johnson R., Zampogni L., Some remarks concerning reflectionless Sturm-Liouville potentials, Stoch. Dyn. 8 (2008), 413-449.

[21] Johnson R., Zampogni L., On the Camassa-Holm and K-dV hierarchies, J. Dynam. Differential Equations 22 (2010), 331-366.

[22] Johnson R., Zampogni L., Remarks on a paper of Kotani concerning generalized reflectionless Schrödinger potentials, Discrete Contin. Dyn. Syst. Ser. B 14 (2010), 559-586.

[23] Johnson R., Zampogni L., The Sturm-Liouville hierarchy of evolution equations, Adv. Nonlinear Stud. 11 (2011), 555-591.

[24] Johnson R., Zampogni L., The Sturm-Liouville hierarchy of evolution equations. II, Adv. Nonlinear Stud. 12 (2012), 501-532.

[25] Johnson R., Zampogni L., On a Gel'fand-Levitan theory for the Sturm-Liouville operator, Preprint.

[26] Knopp K., Funktionentheorie. II. Anwendungen und Weiterführung der allgemeinen Theorie, de Gruyter, Berlin, 1981.

[27] Kotani S., KdV flow on generalized reflectionless potentials, J. Math. Phys. Anal. Geometry 4 (2008), $490-528$.

[28] Lax P.D., Levermore C.D., The small dispersion limit of the Korteweg-de Vries equation. I, Comm. Pure Appl. Math. 36 (1983), 253-290.

[29] Lax P.D., Levermore C.D., The small dispersion limit of the Korteweg-de Vries equation. II, Comm. Pure Appl. Math. 36 (1983), 571-593.

[30] Lax P.D., Levermore C.D., The small dispersion limit of the Korteweg-de Vries equation. III, Comm. Pure Appl. Math. 36 (1983), 809-829.

[31] Levitan B.M., Approximation of infinite-zone by finite-zone potentials, Math. USSR Izv. 20 (1983), 55-87.

[32] Levitan B.M., On the closure of the set of finite-zone potentials, Math. USSR Sb. 51 (1985), 67-89.

[33] Levitan B.M., Inverse Sturm-Liouville problems, VSP, Zeist, 1987.

[34] Lundina D., Compactness of the set of reflectionless potentials, Teor. Funkts. Funkts. Anal. Prilozh. 44 (1985), 55-66.

[35] Marchenko V.A., Sturm-Liouville operators and applications, Operator Theory: Advances and Applications, Vol. 22, Birkhäuser Verlag, Basel, 1986.

[36] Marchenko V.A., The Cauchy problem for the KdV equation with nondecreasing initial data, in What is Integrability?, Springer Ser. Nonlinear Dynam., Springer, Berlin, 1991, 273-318.

[37] Marchenko V.A., Ostrovsky I.V., Approximation of periodic by finite-zone potentials, Sel. Math. Sov. 6 (1987), 101-136.

[38] McKean H.P., The Liouville correspondence between the Korteweg-de Vries and the Camassa-Holm hierarchies, Comm. Pure Appl. Math. 56 (2003), 998-1015.

[39] McKean H.P., van Moerbeke P., The spectrum of Hill's equation, Invent. Math. 30 (1975), 217-274. 
[40] Novikov S., Manakov S.V., Pitaevskiǔ L.P., Zakharov V.E., Theory of solitons. The inverse scattering method, Contemporary Soviet Mathematics, Consultants Bureau, New York, 1984.

[41] Sato M., Soliton equations as dynamical systems on an infinite dimensional Grassmann manifolds, in Random Systems and Dynamical Systems (Kyoto, 1981), RIMS Kokyuroku, Vol. 439, Kyoto, 1981, 30-46.

[42] Segal G., Wilson G., Loop groups and equations of KdV type, Inst. Hautes Études Sci. Publ. Math. (1985), 5-65.

[43] Venakides S., The Korteweg-de Vries equation with small dispersion: higher order Lax-Levermore theory, Comm. Pure Appl. Math. 43 (1990), 335-361.

[44] Zampogni L., On algebro-geometric solutions of the Camassa-Holm hierarchy, Adv. Nonlinear Stud. 7 (2007), 345-380.

[45] Zampogni L., On infinite order K-dV hierarchies, J. Appl. Funct. Anal. 4 (2009), 140-170. 